\title{
Case Report for a Refractory Levator Ani Syndrome Treated with Traditional Korean Medication
}

\author{
Chang-Gue Son \\ Liver and Immunology Research Center, Oriental Medical College in Daejeon University
}

\begin{abstract}
Objectives: To inform a clinical case of a refractory levator ani syndrome, that was improved by treatments of traditional Korean medicine (TKM).

Methods: A 55-years old female had complained severe anorectal pain which didn't respond to Western medicine therapeutics during 5 months including 45 -day hospitalization. Whereas the symptom was rapidly resolved by the treatment in a Korean medicine hospital, and the clinical outcome was monitored.

Result: There was no abnormality explaining the anorectal pain from blood tests, gastrointestinal endoscopy, sonogram and computed tomography for abdomen and pelvis. The patient was diagnosed with a levator ani syndrome. Based on the typical feature of tenderness of lower abdomen as well as beating sign around belly, an herbal drug, Shihogayonggolmoryo-tang (柴胡加龍骨牨蛙湯) was prescribed. In addition, the warm acupuncture at BL 31 to BL 33 and moxibustion on lower abdomen were given to the patient. The anorectal pain was radically reduced from treatment 7-day, and it almost disappeared within treatment 25-day.

Conclusion: This case report would provide information for the potential of TKM therapies focused on the refractory levator ani syndrome which no satisfactory therapy exist.
\end{abstract}

$\overline{\text { Key Words }}$ : Levator ani syndrome, Functional rectal pain, Traditional Korean medicine

\section{Introduction}

Levator ani syndrome (LAS) and proctalgia fugax are variants of functional anorectal pain, which is different in their main region and episodic period of pain ${ }^{1)}$. Comparing to proctalgia fugax, LAS is the more severe form, which presents pain, pressure, and discomfort in the relatively wide region of the rectum, sacrum, and coccyx last over 20 minutes $^{2}$. LAS shows the recurrent or chronic rectal pain, which is precipitated by stress from sitting for long time, childbirth and defecation ${ }^{3)}$.
The etiology of LAS is unknown, but spasm of the levator ani muscles without detectable organic pathology is proposed ${ }^{4)}$. The prevalence of LAS in general population was $6.6 \%$ from a USA national householder survey ${ }^{5)}$. LAS is known to be more common in women, and affects to subjects aged $30^{-}$ 60 years with prevalence declining in subjects over age of 45 years $^{6}$. Patients with the LAS often have psychosocial distress such as depression and anxiety, and impaired quality of life ${ }^{7}$.

No single treatment exists as a successful therapeutics for patients with LAS. There are many options to care the symptoms of LAS, likely digital

\footnotetext{
- Received : 30 May 2017

- Revised : 7 June 2017

- Accepted : 7 June 2017

- Correspondence to : Chang-Gue Son

Liver and Immunology Research Center, Daejeon Oriental Hospital of Daejeon University

176-9, Daeheung-ro, Jung-gu, Daejeon, 34929 Republic of Korea

Tel : +82-42-229-6807, Fax : +82-42-257-6398, E-mail : ckson@dju.ac.kr
} 
massage, sitz baths, diathermy, muscle relaxants and biofeedback therapy respectively ${ }^{8}$. However, some severe cases of patients don't response to above approaches. They suffer from the consistent refractory anorectal pain and symptoms which seriously hurt the daily life.

This study report a case of refractory LAS patient who complained unbearable and unrelieved anorectal pain for 5 months even various therapeutics in Western hospital, but rapidly improved by acupuncture and Shihogayonggolmoryo-tang (柴胡加 龍骨牡奮湯) by traditional Korean medicine-based treatments.

\section{Report of the case}

\section{Characteristics of patient and medical history}

A 55-year-old woman visited a Korean medicine hospital with unbearable anorectal pain persisted for 5 months, which diagnosed with LAS. After getting hemorrhoidectomy in December 2015, her severe anorectal pain started suddenly from January 2016. The pain had been intolerable; therefore the patient had been hospitalized for 45 days in a Western hospital and visited other rectal-specified local clinics. The severity of anorectal pain was not changed, thus she had to lie down for most of day time. The patient hadn't used alcohol, additive drug or smoked, and had no specific past history except only histerectomia due to myoma uteri five years before onset of LAS.

\section{Diagnosis, treatments, and course of symptom}

The patient had been examined thoroughly in a previous Western hospital. From the anoscopy, sigmoidoscopy, intravenous pyelography (IVP), colostogram, pelvic computed tomography (CT), biochemistry, complete blood counts, and urinalysis, no significant abnormality was observed (Fig. 1). The patient was thin (BMI 17.5) and a little tense usually, and lost weight about $4 \mathrm{~kg}$ after LAS-related hospitalization. The status of the coat on her tongue was red color, and her pulsation was weak and rapid. Especially straight muscle of abdomen was very stiff with palpitation in abdominal area, therefore the symptom differentiation was diagnosed as "Congested

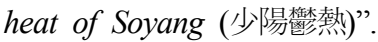

The patient was administrated with an herbal drug, Shihogayonggolmoryo-tang (柴胡加龍骨牡螈湯, Table 2) twice per day. In addition, acupuncture treatment (mainly warm acupuncture at BL 31 to BL 33 daily for 20 min with $0.25 \times 30$ needles purchased from DongBang Co. Seoul), indirect moxibustion on lower abdomen were given to the patients. The anorectal pain began to decrease from treatment 3-day and notably by 5 points by self-reporting numerical rating scale (NRS, 0 means no pain while 10 doses the worst severity ${ }^{99}$ ) on treatment 12-day respectively. The anorectal pain as well as stiffness and palpitation in abdomen were almost disappeared within 25 days, and she could do daily house works (Fig. 2). She had gained body weight about $1 \mathrm{~kg}$ during treatment with acupuncture and herbal drug. It was confirmed that the patient has been healthy in general via telephone interview thereafter.

\section{Discussion and Conclusion}

The case reported herein presented a refractory LAS of the 55-year-old woman. Her symptom of LAS suddenly started on one month later hemorrhoidectomy, which was unbearable anorectal pain. Even though the patient got many examinations and therapeutics including muscle relaxants and anodynia as inpatient or outpatient, the anorectal pain was not changed for 5 months.

The anorectal pain however had been relieved rapidly by warm acupuncture at BL 31 to BL 33, indirect moxibustion and administration with 

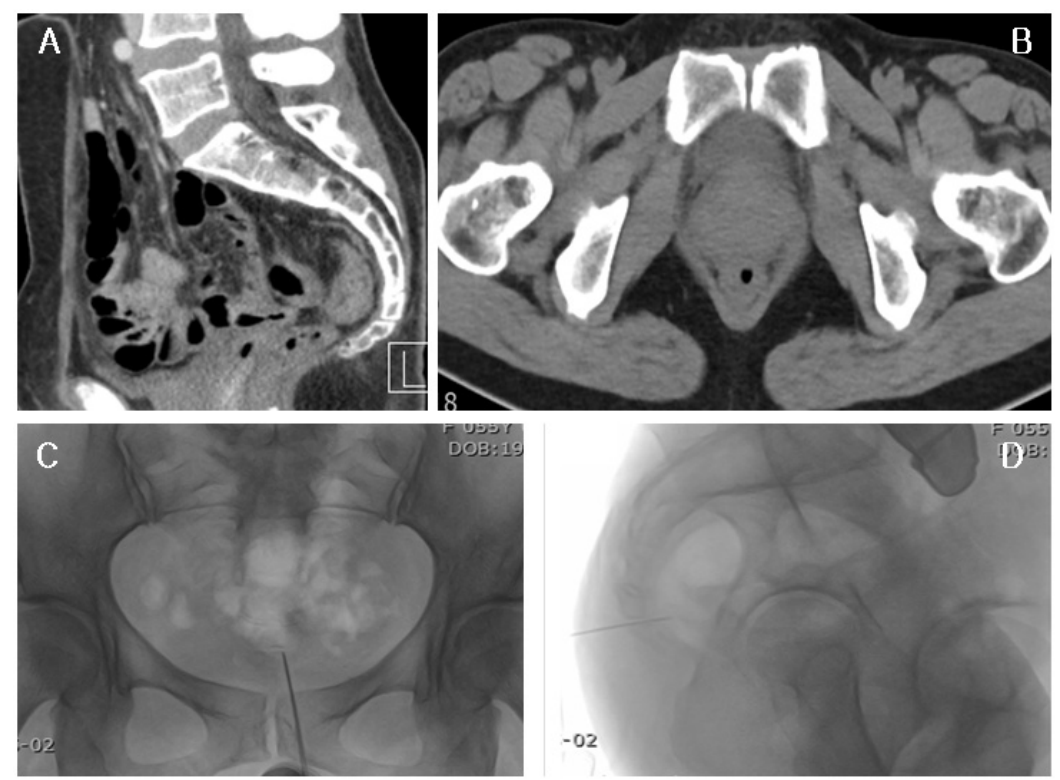

Fig. 1. Pelvic CT (A,B), IVP (C) and colostogram (D). No abnormality was found in radiologic exam inations.

A

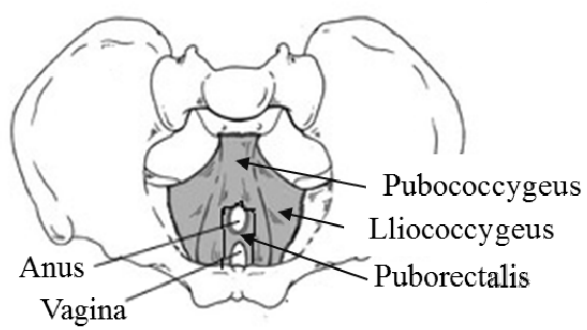

B

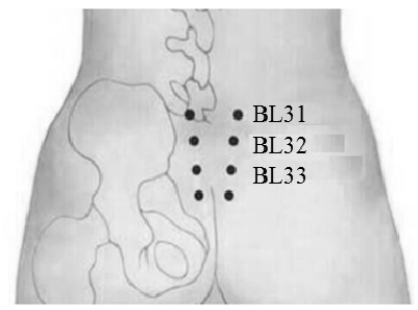

Fig. 2. Levator ani muscles (A) and Acupoints (BL31 to BL33)

2010 : Histerectomia

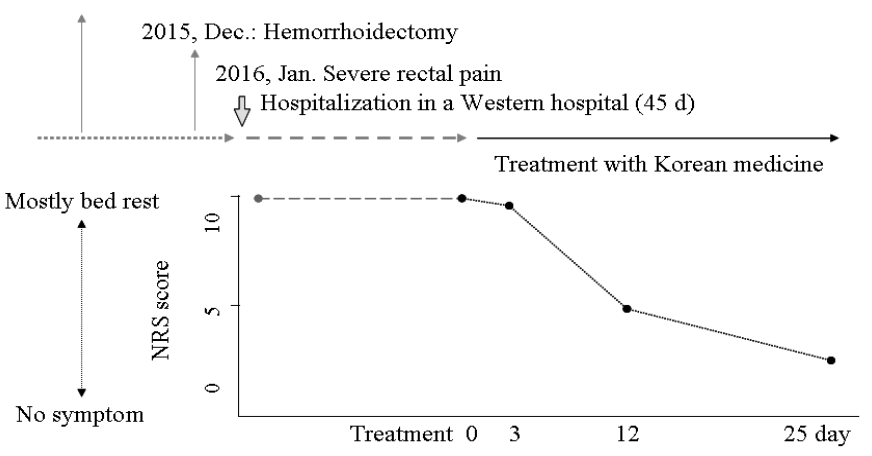

Fig. 3. Summary of clinical courses and treatment outcome 
Shihogayonggolmoryo-tang (柴胡加龍骨牡蠇湯). These choices of treatments were decided based on the diagnosis by Korean medicine system. The warm acupuncture is the combination therapy of acupuncture and moxibustion, which is applied to disorders associated with cold symptom and chronic pain ${ }^{10)}$. The chronic pain in low abdominal and pelvic area is generally caused by cold and stagnation of Qi. Acupoints of BL 31 to BL 33 is known to be effective to pelvic disorders ${ }^{11)}$. The herbal drug, Shihogayonggolmoryo-tang (柴胡加龍骨 牡蜮湯) is prescribed to treat patients suffering from neurasthenia including palpitation, insomnia, or frequent anger ${ }^{12)}$. In particular the current choice of Shihogayonggolmoryo-tang (柴胡加龍骨牡蠣湯) was relied on the stiff pattern of abdominal muscle with palpitation, which differentiated as "Congested heat of Soyang (少陽鬱熱)”. Shihogayonggolmoryo-tang (柴胡加龍骨牡蠇湯) was well known to be effective on the case of above abdominal stiff with palpitation and neurotic status ${ }^{13,14)}$.

Levator ani muscles are formed from three muscle components: the puborectalis, pubococcygeus and iliococcygeus muscle, which are situated on either side of the pelvis and supports the viscera in the pelvic cavity ${ }^{15}$. The spasm of these muscles is thought as a main cause of LAS, but one group found a tendency of hyper-contractile external anal sphincter than spasm in LAS comparing to proctalgia fugax ${ }^{16)}$. The biofeedback therapy or the combination of several approaches such as digital massage, sitz baths, diathermy, and muscle relaxants, can reduce the anorectal pain in most of patients with LAS $^{17}$. Some cases however, complain the chronic and persistent pain leading to the impaired seriously quality of life, and then those refractory cases were more frequently happen in patients of neurotic personality likely the present patient ${ }^{18)}$. To date there is less of academic report for treatment of LAS using TKM even though many advertisements for LAS are shown by Oriental clinics, and one group presented a refractory case of LAS treated by acupuncture in $\mathrm{USA}^{19}$.

In summary, in spite of limitation of only a case of LAS, this study presents that TKM-based strategy could be effective for refractory LAS. Further clinical observations and applications would need to develop the confirmative therapeutics for LAS in the future.

\section{Acknowledgement}

This research was supported by the grant of the Traditional Korean Medicine R\&D Project, Ministry of Health \& Welfare, South Korea (HI16C0112 -000015).

\section{References}

1. Bharucha AE, Lee TH. Anorectal and Pelvic Pain. Mayo Clin Proc. 2016;91(10):1471-86.

2. Ng CL. Levator ani syndrome - a case study and literature review. Aust Fam Physician. 2007;36(6):449-52.

3. Wald A. Functional anorectal and pelvic pain. Gastroenterol Clin North Am. 2001;30:243-51.

4. Hull M, Corton MM. Evaluation of the levator ani and pelvic wall muscles in levator ani syndrome. Urol Nurs. 2009;29(4):225-31.

5. Drossman DA, Li Z, Andruzzi E, Temple RD, Talley NJ, Thompson WG, et al. U.S. householder survey of functional gastrointestinal disorders. Prevalence, sociodemography, and health impact. Dig Dis Sci. 1993;38(9):1569-80.

6. Whitehead WE, Wald A, Diamant NE, Enck P, Pemberton JH, Rao SSC. Functional disorders of the anus and rectum. Gut. 1999;45(Suppl 2):55-9.

7. Potts JM, Payne CK. Urologic chronic pelvic pain. Pain. 2012;153(4):755-8.

8. Grant SR, Salvati EP, Rubin RJ. Levator syndrome: an analysis of 316 cases. Dis Colon Rectum. 1975;18(2):161-3. 
9. Hartrick CT, Kovan JP, Shapiro S. The numeric rating scale for clinical pain measurement: a ratio measure? Pain Pract. 2003;3(4):310-6.

10. An TEB, Kim DC. Clinical Study for Eight Cases of Dysmenorrhea Treated by Sobokchukeo -tang-gagambang and Warm Needle Therapy. J Ori Obstetrics and Gynecol. 2016;29(3):57-67.

11. Jang MW, Kwon YJ, Jo WK, Jung SH, Bae HS, Lim SW, et al. Two Cases of Voiding Problem Treated with Oriental Medicine. Korean J. Oriental Phy \& Path. 2012;26(2):241-7.

12. Kim KS, Kim WC, Kim KO. A Study about trends of using Shihogayonggolmoryo-tang granule. J. Herbal Formula Science. 2012;20(1): 159-66.

13. Seo SH, Yim HJ, Jung IC, Lee SY. The clinical study on 1 case of Patient with Tremor by Bok-Jin. J Korean medicine institute of Daejeon University. 2007;16(1):69-79.

14. Lee YH. 5 Cases Report of Climacteric Symptoms with Shihogayonggolmoryo-tang. J Ori Obstetrics and Genecol. 2013;26(1):121-34

15. Tsukada $\mathrm{Y}$, Ito $\mathrm{M}$, Watanabe $\mathrm{K}$, Yamaguchi $\mathrm{K}$, Kojima M, Hayashi R, et al. Topographic
Anatomy of the Anal Sphincter Complex and Levator Ani Muscle as It Relates to Intersphincteric Resection for Very Low Rectal Disease. Dis Colon Rectum. 2016;59(5):426-33.

16. Jang GY, Lee JS, Lim HH, Hwang KR, Hong SJ, Kim JO, et al. Anorectal Symtoms and Anorectal Pathophysiologic Findings in Patients with Levator Ani Syndrome and Protalgia Fugax. Korean J Neurogastroenterology and Motility. 2003:9(1):37-41.

17. Chiarioni G, Nardo A, Vantini I, Romito A, Whitehead WE. Biofeedback is superior to electrogalvanic stimulation and massage for treatment of levator ani syndrome. Gastroenterology. 2010;138(4):1321-29.

18. Bharucha AE, Trabuco E. Functional and chronic anorectal and pelvic pain disorders. Gastroenterol Clin North Am. 2008;37(3): 685-96.

19. Saleemi HT, Farooque H, Choudhury S, Zhang J. Acupuncture Treatment for Levator Ani Syndrome: A Case Report. PM\&R. 2016;8(9S): S169. 